\title{
IDENTIFICACIÓN Y VALORACIÓN DE LOS PUNTOS DE INTERÉS DIDÁCTICO DEL MUNICIPIO DE ÁGUILAS
}

\author{
Juan Carlos Escaravajal Rodríguez ${ }^{1}$, Juana Ester Blázquez Perán ${ }^{2}$ \\ Universidad de Granada y Universidad de Murcia
}

\begin{abstract}
RESUMEN
El objetivo del presente trabajo es identificar y valorar los puntos de interés didáctico turístico existentes en Águilas. En cuanto a la metodología, mediante un diseño observacional, se ha utilizado el método cualitativo a través de una hoja de observación, para valorar diversos factores asociados a los puntos de interés. La muestra está compuesta por 59 bienes patrimoniales, y para el análisis de los datos se ha realizado una estadística descriptiva de frecuencias y porcentajes. En base a los resultados obtenidos, se puede concluir que Águilas posee al menos 59 puntos de interés, éstos se encuentran en un estado de conservación principalmente desfavorable, la mayoría puede visitarse sin inconvenientes, un importante porcentaje no es accesible para personas con movilidad reducida y, además, la mayoría no posee información "in situ". A partir de estos datos se pueden iniciar y valorar diversas propuestas para facilitar el acercamiento del patrimonio a los ciudadanos.
\end{abstract}

Palabras clave: patrimonio cultural; hoja de observación; valoración; guía; puntos de interés didáctico.

\section{IDENTIFICATION AND ASSESSMENT OF DIDACTIC POINTS OF INTEREST IN THE MUNICIPALITY OF ÁGUILAS}

\begin{abstract}
The aim of this project is to identify and assess every existent didactic point of interest in Águilas. Regarding the methodology, using an observational design, a qualitative method through an observation sheet has been employed; in order to assess different factors associated to these points of interest. The sample is made of 59 heritage assets and, for the data analysis, a descriptive statistics of frequency and percentages were created. According to the results, it can be concluded that Águilas has at least 59 points of interest, these are now in an unfavorable condition, and although the majority of them can be visited without any inconvenient, a significant percentage is not accessible to people with reduced mobility and moreover, the majority of them does not have on-site information. From this data we can start and assess several proposals in order to bring this heritage closer to the citizens
\end{abstract}

Key words: cultural heritage; observation sheet; assessment; guide; didactic points of interest.

\footnotetext{
1 Facultad de Ciencias de la Educación, Universidad de Granada. E-mail: jcescaravajalrodriguez@gmail.com

${ }^{2}$ Facultad de Educación, Universidad de Murcia .E-mail: juaniesther_93@hotmail.com
}

Fecha de recepción: 22 de septiembre de 2019. Fecha de aceptación: 26 de diciembre de 2019

Papeles de Geografía, 65 (2019), 121-138 


\section{INTRODUCCIÓN}

El patrimonio histórico español está integrado por los bienes inmuebles y muebles de interés artístico, arqueológico, etnográfico, histórico, paleontológico, técnico o científico. Además del patrimonio documental y bibliográfico, las zonas arqueológicas y yacimientos, sitios naturales, parques y jardines, que posean un valor artístico, antropológico o histórico (JEFATURA DEL ESTADO, 29 de junio de 1985). Así, el patrimonio es un referente para la formación de identidades culturales por parte de las estructuras sociales y los ciudadanos (CUENCA, 2014) a lo largo del tiempo. A través de la interpretación del patrimonio histórico se pueden conocer y entender las culturas pasadas, y mediante la observación de los elementos patrimoniales activos como pueden ser naturales, etnológicos, científicos, etc., se puede determinar la identidad cultural del lugar en el presente.

Por lo tanto, se considera como patrimonio cultural al conjunto de bienes tangibles e intangibles creados por el ser humano y que expresan sus identidades culturales, su historia, etc., reconociéndose así los parques naturales, costumbres y leyendas, artesanía, obras de ingeniería, entre otros elementos que conforman el repertorio patrimonial (MAILLARD, 2012).

Así mismo, la UNESCO (2014, p.134) determina como patrimonio cultural los siguientes elementos:

- Los monumentos: obras arquitectónicas, de escultura o de pintura monumental, elementos o estructuras de carácter arqueológico, inscripciones, cavernas y grupos de elementos, que tengan un valor universal excepcional desde el punto de vista de la historia, del arte o de la ciencia.

- Los conjuntos: grupos de construcciones, aisladas o reunidas, cuya arquitectura, unidad e integración en el paisaje les dé un valor universal excepcional desde el punto de vista de la historia, del arte o de la ciencia;

- Los lugares: obras del hombre u obras conjuntas del hombre y la naturaleza, así como las zonas, incluidos los lugares arqueológicos, que tengan un valor universal excepcional desde el punto de vista histórico, estético, etnológico o antropológico.

La valoración de los elementos patrimoniales no siempre depende de ellos, como el peso o la forma, sino que también depende de una cualidad subjetiva, asociada a la percepción de las personas, que varía en función de cada una y que al final atribuirán un valor (BALLART, 2001), y de ello dependerá el uso que se les dé, ya sea científico, turístico o didáctico.

Por otro lado, algunos de los bienes que configuran el entorno pueden estar sujetos a la Ley del Patrimonio Histórico Español (JEFATURA DEL ESTADO, 29 de junio de 1985), la cual establece distintos niveles de protección e integra aquellos bienes de valor histórico, científico, artístico o técnico. Existiendo, con el objetivo de otorgar una mayor protección y amparo, la categoría de bienes de interés cultural (BIC) que se extiende a los muebles e inmuebles del Patrimonio que requiere una especial protección. Seguida de otras figuras asignadas por las comunidades autónomas, por ejemplo, en nuestro caso, en la Región de Murcia se establecen las siguientes categorías para los bienes más destacadas del patrimonio cultural de dicha comunidad autónoma (COMUNIDAD AUTÓNOMA DE LA REGIÓN DE MURCIA, 12 de abril de 2007):

- Bienes de interés cultural: figura asignada a los bienes muebles, inmuebles e inmateriales más relevantes por su sobresaliente valor cultural para la Región de Murcia.

- Bienes catalogados por su relevancia cultural: figura asignada a los bienes muebles, inmuebles e inmateriales que posean una notable relevancia cultural, pero que no merecen la protección derivada de BIC.

- Bienes inventariados: figura asignada a los bienes culturales que, pese a su destacado valor cultural, no merecen la protección de BIC o bienes catalogados por su relevancia cultural.

A su vez, el Ayuntamiento de Águilas clasifica estos bienes de acuerdo a los siguientes grados de protección, ordenados de mayor a menor tipo de conservación de sus elementos arquitectónicos (AYUNTAMIENTO DE ÁGUILAS, s.f.a): protección integral (grado 1), protección parcial (grado 2) y protección parcial (grado 3).

Por otro lado y en el caso del medio natural, éste constituye un espacio geográfico diverso y heterogéneo, con multitud de elementos característicos que dan lugar a una gran variedad de 
paisajes (MÍNGUEZ, 2010; TORT, 2004). Algunos de estos parajes pueden poseer la figura de espacios naturales protegidos (ENP), siendo considerados como los principales entornos para el desarrollo de programas y estrategias de trabajo, ofreciendo oportunidades para la realización de actividades con fines didácticos, sobre todo en aquellos espacios que cuenten con una red de equipamientos bien articulada (SERRANO DE LA CRUZ, GARCÍA y JEREZ, 2017).

Más allá y establecido por la Ley Orgánica 16/2007 (JEFATURA DEL ESTADO, 14 de diciembre de 2007), los ENP se clasifican en función de los bienes y valores a proteger, y de los objetivos de gestión a cumplir, encontrando: parques, reservas naturales, áreas marinas protegidas, monumentos naturales y paisajes protegidos. Por otro lado, además de las anteriores designaciones, los espacios naturales podrán estar integrados dentro de la Red Ecológica Europea Natura 2000 y poseer las siguientes figuras: lugares de importancia comunitaria (LIC), las zonas especiales de conservación (ZEC) y las zonas de especial protección para las aves (ZEPA).

A través de la didáctica de los bienes patrimoniales existentes se puede potenciar el conocimiento reflexivo de la realidad, así como el conocimiento de los propios elementos patrimoniales y sus procesos de análisis e investigación (CUENCA, ESTEPA y MARTÍN, 2011).

Los puntos de interés didáctico (PIDs) se pueden entender como enclaves naturales o culturales, cuya singularidad y características facilitan el conocimiento "in situ" de sus valores científicos y/o culturales, a través de un enfoque pedagógico (CORVEA, 2001). Estos elementos pueden ser percibidos por los visitantes, ya que destacan en el entorno, pero muchas veces no nos percatamos de las posibilidades didácticas que poseen (CORVEA, BUSTAMANTE, GARCÍAHIDALGO, SANZ y MATEOS, 2006), siendo útiles para trabajar contenidos curriculares en la educación formal y en la no formal, ya que pueden aportar un conocimiento enriquecedor a sus visitantes a la vez que promover su valor sociabilizador.

Estos PIDs pueden ser visitados conjuntamente a través de un itinerario, el cual puede ser definido como "un recorrido o camino a seguir con diferentes puntos de parada o de interés en determinados elementos de valor patrimonial o cultural" (INSA, 2002, p. 89), convirtiéndose en una herramienta ideal para fomentar el conocimiento del patrimonio de la zona de interés. Siendo por ello necesario analizar, previamente, la situación actual de los bienes patrimoniales del municipio en cuestión, entendiendo éstos como elementos educativos para acercar al ciudadano (puntos de interés didáctico).

En el ámbito de la educación formal, este tipo de actividades enriquece el aprendizaje del alumnado: gran motivación, desarrollo del vocabulario, acercamiento a lo real, aprendizaje mediante la observación, capacidad de analizar, comprender y desarrollar las destrezas, capacidad crítica y desarrollo de valores éticos (GARCÍA, 1994).

Atendiendo al currículo de la Educación Primaria en la Región de Murcia (CONSEJO DE GOBIERNO, 06 de septiembre de 2014), los itinerarios didácticos, así como sus puntos de interés didáctico, se relacionan directamente con diversos contenidos establecidos en las áreas de las Ciencias de la Naturaleza, las Ciencias Sociales y la Educación Física. En relación al currículo de la Educación Secundaria Obligatoria en la Región de Murcia (CONSEJO DE GOBIERNO, 03 de septiembre de 2015a) se relacionan directamente con diversos contenidos establecidos en las materias de la Biología y la Geología, la Geografía e Historia, y la Educación Física. Y por último, atendiendo al currículo de la etapa de Bachillerato en la Región de Murcia (CONSEJO DE GOBIERNO, 03 de septiembre de 2015b) se relacionan directamente con diversos contenidos establecidos en las materias de Biología y Geología, Geografía, Geología, Ciencias de la Tierra y del Medioambiente, Historia del Arte, Fundamentos del Arte, y la Educación Física.

Además, en relación a las competencias (MINISTERIO DE EDUCACIÓN, CULTURA Y DEPORTE, 29 de enero de 2015), los itinerarios didácticos y PIDs contribuyen al desarrollo de:

- Comunicación lingüística: el uso del lenguaje se manifiesta de varias formas: lectura (en la búsqueda de información), escrita (en la cumplimentación del cuaderno) y expresión oral (en las interacciones con los compañeros).

- Competencia matemática y competencias básicas en ciencia y tecnología: fundamentalmente se fomenta la segunda competencia, ya que con los itinerarios se abordarán contenidos relacionados con las Ciencias Sociales y Naturales, como el patrimonio histórico, la flora, la fauna, etc. 
- Competencia digital: ésta se puede fomentar en el caso de integrar las tecnologías en el itinerario didáctico, a través del uso del teléfono móvil, códigos QR, aplicaciones de realidad aumentada, etc.

- Aprender a aprender: el alumno debe explorar y buscar la información solicitada de los puntos de interés por el profesorado o el cuaderno, aprendiendo a saber la que es correcta o útil.

- Competencias sociales y cívicas: este tipo de actividades se suelen realizar en parejas o grupos pequeños, por lo que resulta imprescindible la coordinación y buena armonía entre los compañeros. Además, la aportación de cada uno ellos dará lugar al resultado final. Y por último, los itinerarios promueven el vínculo del alumno con su ciudad y su entorno.

- Conciencia y expresiones culturales: con este tipo de actividades se favorece el conocimiento del patrimonio histórico, cultural y/o natural, fomentando a la vez el respeto hacia el mismo.

En esta línea, se pueden encontrar diversos trabajos donde se desarrollan itinerarios didácticos previa selección de los PIDs, por ejemplo: en el Parque Nacional de la Sierra de Guadarrama (CRESPO, GÓMEZ y CRUZ, 2018), zona norte de la Comunidad de Madrid (CORVEA, 2001; CORVEA et al., 2006), varios espacios de la provincia de Almería (LÓPEZ y SEGURA, 2013), término municipal de Patones (Comunidad de Madrid) (MARTÍN, 2016), municipio de Fuente Álamo (Región de Murcia) (MARTÍNEZ y GARCÍA, 2008), siendo una práctica común para la puesta en valor del patrimonio natural y cultural existente en nuestro entorno próximo, desde la escuela con el alumnado hasta en el tiempo de ocio de los ciudadanos.

En el caso del presente trabajo, Águilas es el municipio objeto, el cual pertenece a la comarca del Alto Guadalentín (Región de Murcia). Limita al norte y este con el término municipal de Lorca, al sur con el Mar Mediterráneo y al oeste con el municipio almeriense de Pulpí. Además, posee una costa de 28 kilómetros, así como varios espacios naturales como el Parque Regional de Cabo Cope-Calnegre y el Paisaje Natural de Cuatro Calas (Región de Murcia Digital, s. f.). Por lo que posee un amplio territorio costero y de interior con una gran variedad de patrimonio cultural (AYUNTAMIENTO DE ÁGUILAS, s.f.a).

Por todo ello, el objeto del presente trabajo es identificar y valorar los puntos de interés didáctico y turístico existentes en Águilas.

\section{METODOLOGÍA}

\subsection{MÉTODO}

En la presente investigación, mediante un diseño observacional, se ha utilizado el método cualitativo a través de una hoja de observación para la valoración de diversos factores asociados a los puntos de interés didáctico seleccionados.

\subsection{MUESTRA}

La muestra de esta investigación estuvo compuesta por 59 PIDs, los cuales fueron seleccionados por ser parte del patrimonio cultural e histórico de Águilas.

\subsection{TÉCNICAS E INSTRUMENTOS}

Para la obtención de información relacionada con los PIDs se ha realizado una revisión bibliográfica de material publicado sobre el territorio objeto, como mapas, informes, comunicados, catálogos, etc. Posteriormente, los PIDs han sido analizados utilizando una hoja de observación "ad hoc" (tabla 1), adaptada de MARTÍN (2016) y compuesta seis indicadores: a) estado de conservación, b) estacionalidad, c) condiciones de observación, d) peligrosidad del terreno, e) accesibilidad y f) información disponible.

Hoja de observación de los PIDs 


\begin{tabular}{|c|c|}
\hline Indicador & Categoría y descripción \\
\hline $\begin{array}{l}\text { Estado de } \\
\text { conservación }\end{array}$ & $\begin{array}{l}\text { Fuertemente degradado: Se encuentra prácticamente destruido o con } \\
\text { deterioros importantes. } \\
\text { Alterado: Se encuentra con deterioros que impiden apreciar algunas } \\
\text { características de interés. } \\
\text { Favorable con alteración: presenta algunos deterioros que no afectan de } \\
\text { manera determinante al valor o interés del PID. } \\
\text { Favorable: El PID se encuentra bien conservado, prácticamente íntegro. }\end{array}$ \\
\hline Estacionalidad & $\begin{array}{l}\text { El lugar no puede visitarse por restricciones legales. } \\
\text { El lugar puede visitarse puntualmente a lo largo del año. } \\
\text { El lugar puede visitarse en cualquier época del año, pero varían sus } \\
\text { características y lo hacen menos propicio en determinados periodos. } \\
\text { El lugar puede visitarse, teniendo en cuenta que se encuentra en un } \\
\text { entorno privado. } \\
\text { El lugar puede visitarse en cualquier época del año, teniendo en cuenta } \\
\text { el horario establecido de visita o sin horario. }\end{array}$ \\
\hline $\begin{array}{l}\text { Condiciones de } \\
\text { observación }\end{array}$ & $\begin{array}{l}\text { Presencia de elementos que enmascaran fuertemente las características } \\
\text { de interés. } \\
\text { Presencia de elementos que enmascaran el PID y que impiden apreciar } \\
\text { algunas características de interés. } \\
\text { Con algún elemento que impide observar el PID en su integridad pero } \\
\text { que no afectan a la apreciación de sus características de interés. } \\
\text { Perfectamente observable en su integridad y con facilidad. }\end{array}$ \\
\hline $\begin{array}{l}\text { Peligrosidad del } \\
\text { terreno }\end{array}$ & $\begin{array}{l}\text { Existen elementos que hacen que el lugar sea altamente peligroso, aun } \\
\text { tomando medidas de seguridad y precaución. } \\
\text { Existen elementos que hacen del lugar parcialmente peligroso, aun } \\
\text { tomando medidas de seguridad. } \\
\text { Existen elementos que hace que el lugar sea parcialmente peligroso solo } \\
\text { si no se toman medidas de seguridad. } \\
\text { No existe peligrosidad asociada al lugar. }\end{array}$ \\
\hline Accesibilidad & $\begin{array}{l}\text { Sin acceso directo por camino transitable. } \\
\text { Con acceso directo por camino transitable para personas que no } \\
\text { presentan movilidad reducida. } \\
\text { Con acceso directo por camino transitable para personas con movilidad } \\
\text { reducida y resto de usuarios. }\end{array}$ \\
\hline $\begin{array}{l}\text { Información } \\
\text { disponible }\end{array}$ & $\begin{array}{l}\text { El PID no posee ningún tipo de información "in situ". } \\
\text { El PID posee información "in situ". }\end{array}$ \\
\hline
\end{tabular}

Fuente: adaptado de MARTíN (2016)

\subsection{PROCEDIMIENTO DE RECOGIDA DE INFORMACIÓN}

Para la recogida y selección de los datos de los PIDs se utilizó una adaptación de la metodología establecida por CORVEA (2001), fundamentada en cuatro etapas:

1. Estudio previo e inventario. Análisis y revisión bibliográfica de documentos y materiales publicados sobre el territorio, tanto natural como urbano (mapas, informes, comunicaciones, catálogos, esquemas, etc.), además de la información que puedan facilitar docentes e investigadores que tengan conocimiento del entorno analizado. En esta fase se han consultado diversos documentos: catálogo etnográfico (AYUNTAMIENTO DE ÁGUILAS, s.f.a), catálogo de edificios protegidos (AYUNTAMIENTO DE ÁGUILAS, s.f.b), catálogo arqueológico y paleontológico (AYUNTAMIENTO DE ÁGUILAS, s.f.c), catálogo de árboles singulares y monumentales (AYUNTAMIENTO DE ÁGUILAS, s.f.d), y documento III, catálogo de 
elementos protegidos (AYUNTAMIENTO DE ÁGUILAS, s.f.e). Finalmente, debido a la inmensa cantidad de puntos existentes en los diversos catálogos, se ha decidido seleccionar únicamente los puntos pertenecientes al catálogo etnográfico y al de edificios protegidos, además de seleccionar los PIDs no catalogados y existentes en el entorno urbano de Águilas, debido a su cercanía en el día a día de los ciudadanos.

2. Descripción. Tras la ubicación del punto, utilizando la geolocalización, se ha precisado el tipo de observación y acceso, y se ha descrito las características e historia del elemento, añadiendo asimismo una foto para la visualización de éste. Por otro lado, se ha colocado un código $\mathrm{QR}$ con redirección a la geolocalización de la ubicación del punto y otro QR con la información leída a través de un audio.

3. Clasificación. En esta fase se ha tenido en cuenta: las figuras asignadas al elemento y al entorno, y las áreas educativas relacionadas directamente.

- Figuras asignadas: bien de interés cultural, bienes catalogados por su relevancia cultural y bienes inventariados, además del grado (1,2 y 3$)$

- Figuras asignadas al entorno: espacios naturales protegidos, Red Natura 2000, lugares de importancia comunitaria, zonas especiales de conservación y zona de especial protección para las aves.

- Áreas educativas:

- Primaria: Ciencias de la Naturaleza, y Ciencias Sociales.

- Secundaria: Biología y Geología, y Geografía e Historia.

- Bachillerato: Biología y Geología, Geografía, Geología, Ciencias de la Tierra y del Medio Ambiente, Fundamentos del Arte, e Historia del Arte.

Además, en esta fase se ha utilizado la hoja de observación (tabla 1), formada por indicadores que ofrecen información que ayudará a determinar la adecuación de las visitas. Para ello, dos observadores, previa práctica y entrenamiento, se desplazaron a cada uno de los PIDs y rellenaron la correspondiente hoja de observación. Se comprobó la calidad del dato con el cálculo del grado de concordancia o fiabilidad del registro de los dos observadores, utilizando el índice Kappa de Cohen, en el que se obtuvo un valor de 1 en la fiabilidad interobservadores. Además, para la fiabilidad intraobservador, los dos observadores analizaron la misma muestra en dos momentos diferentes, dos semanas de diferencia, obteniendo un $100 \%$ de concordancia entre ambas observaciones analizas.

4. Inventario cartográfico. Por último, a partir de un mapa base del área estudiada, se ha confeccionado un mapa de recursos didácticos en la plataforma MyMaps. En él se ha representado los PIDs organizados por la figura de protección asignada y ordenados alfabéticamente, de forma que facilite su visualización.

Tras finalizar los cuatro pasos anteriores, se creó la ficha de los PIDs pertenecientes a la futura guía de puntos de interés didáctico, además de realizar un análisis cuantitativo de los indicadores y clasificaciones asignadas.

\subsection{ANÁLISIS DE DATOS}

Los datos obtenidos han sido examinados a través de un análisis cuantitativo con el software Statistical Package for Social Sciences 20.0 (SPSS-20.0), con el cual se realizó una estadística descriptiva de frecuencias y porcentajes. Además, para comprobar la fiabilidad del registro intraobservador e interobservadores se ha utilizado el índice Kappa de Cohen.

\section{RESULTADOS}


Tras recopilar los puntos recogidos en el catálogo etnográfico (AYUNTAMIENTO DE ÁGUILAS, s.f.a) y el catálogo de edificios protegidos (AYUNTAMIENTO DE ÁGUILAS, s.f.b), además de seleccionar los PIDs no catalogados y existentes en el entorno urbano de Águilas, finalmente se obtuvo un total de 59 PIDs para analizar (Tabla 2).

TABLA 2

Relación de PIDs por protección asignada

\begin{tabular}{|c|c|c|c|c|c|}
\hline \multirow{2}{*}{$\begin{array}{l}\text { Bien de } \\
\text { interés } \\
\text { cultural }\end{array}$} & \multicolumn{4}{|c|}{ Bienes catalogados por su relevancia cultural } & \multirow{3}{*}{$\begin{array}{c}\begin{array}{c}\text { Otros bienes } \\
\text { sin figura }\end{array} \\
\text { Auditorio y } \\
\text { palacio de } \\
\text { congresos } \\
\text { "Infanta } \\
\text { Doña Elena" }\end{array}$} \\
\hline & \multicolumn{2}{|c|}{ Grado 1} & \multirow{2}{*}{\begin{tabular}{c} 
Grado 2 \\
\multicolumn{1}{c}{ Casa } \\
colonial del \\
Huerto Don \\
$\quad$ Jorge
\end{tabular}} & \multirow{2}{*}{$\begin{array}{c}\text { Grado } 3 \\
\text { Antiguo } \\
\text { hospital de } \\
\text { San } \\
\text { Francisco }\end{array}$} & \\
\hline $\begin{array}{l}\text { Castillo de } \\
\text { Chuecos }\end{array}$ & $\begin{array}{l}\text { Acueducto- } \\
\text { dique } \\
\text { Minglano }\end{array}$ & $\begin{array}{l}\text { Ermita de la } \\
\text { Cuesta de } \\
\text { Gos }\end{array}$ & & & \\
\hline $\begin{array}{l}\text { Castillo de } \\
\text { San Juan de } \\
\text { las Águilas }\end{array}$ & $\begin{array}{l}\text { Acueducto de } \\
\text { los } \\
\text { Melenchones }\end{array}$ & $\begin{array}{l}\text { Ermita de } \\
\text { San Isidro }\end{array}$ & $\begin{array}{c}\text { Casa } \\
\text { consistorial } \\
\text { y su fachada }\end{array}$ & $\begin{array}{c}\text { Faro de } \\
\text { Punta Negra }\end{array}$ & $\begin{array}{c}\text { Campo de } \\
\text { fútbol del } \\
\text { Rubial }\end{array}$ \\
\hline $\begin{array}{l}\text { Castillo de } \\
\text { Tébar }\end{array}$ & $\begin{array}{l}\text { Acueducto } \\
\text { del } \\
\text { Labradorcico }\end{array}$ & $\begin{array}{l}\text { Ermita y casa } \\
\text { de Chuecos }\end{array}$ & $\begin{array}{l}\text { Plaza de } \\
\text { Abastos }\end{array}$ & $\begin{array}{l}\text { Iglesia del } \\
\text { Carmen }\end{array}$ & $\begin{array}{l}\text { Casa de la } \\
\text { cultura } \\
\text { Francisco } \\
\text { Rabal }\end{array}$ \\
\hline $\begin{array}{l}\text { Embarcadero } \\
\text { del Hornillo }\end{array}$ & $\begin{array}{l}\text { Acueducto } \\
\text { Monte } \\
\text { Nacimiento }\end{array}$ & $\begin{array}{l}\text { Estación y } \\
\text { museo del } \\
\text { ferrocarril }\end{array}$ & & $\begin{array}{l}\text { Molino de } \\
\text { los } \\
\text { Alacranes }\end{array}$ & $\begin{array}{c}\text { Centro de } \\
\text { interpretación } \\
\text { del mar } \\
\text { (CIMAR) }\end{array}$ \\
\hline $\begin{array}{l}\text { Ermita de } \\
\text { Cope }\end{array}$ & $\begin{array}{c}\text { Acueducto y } \\
\text { pilica Casa de } \\
\text { la Tejera }\end{array}$ & $\begin{array}{c}\text { Ficus } \\
\text { centenarios }\end{array}$ & & $\begin{array}{l}\text { Molino de } \\
\text { Sagrera }\end{array}$ & Ícaro \\
\hline $\begin{array}{l}\text { Isla del } \\
\text { Fraile }\end{array}$ & $\begin{array}{c}\text { Acueducto y } \\
\text { pilica de Casa } \\
\text { Aragón }\end{array}$ & $\begin{array}{c}\text { Iglesia } \\
\text { parroquial del } \\
\text { Garrobillo }\end{array}$ & & $\begin{array}{c}\text { Museo } \\
\text { arqueológico }\end{array}$ & $\begin{array}{c}\text { Monumento } \\
\text { a Francisco } \\
\text { Rabal }\end{array}$ \\
\hline $\begin{array}{l}\text { Molino de } \\
\text { Cope }\end{array}$ & $\begin{array}{l}\text { Acueducto y } \\
\text { pilica de las } \\
\text { Escalericas }\end{array}$ & $\begin{array}{l}\text { Iglesia de } \\
\text { San José }\end{array}$ & & & $\begin{array}{l}\text { Museo del } \\
\text { fútbol } \\
\text { aguileño }\end{array}$ \\
\hline $\begin{array}{c}\text { Puente de las } \\
\text { Culebras }\end{array}$ & $\begin{array}{c}\text { Búnker } \\
\text { Calarreona }\end{array}$ & $\begin{array}{l}\text { Molino del } \\
\text { Saltaor }\end{array}$ & & & $\begin{array}{l}\text { Peñón del } \\
\text { Roncaor }\end{array}$ \\
\hline $\begin{array}{c}\text { Torre de } \\
\text { Cope }\end{array}$ & $\begin{array}{c}\text { Búnker } \\
\text { Matalentisco I }\end{array}$ & $\begin{array}{l}\text { Monumento } \\
\text { al ferrocarril }\end{array}$ & & & $\begin{array}{c}\text { Pico de } \\
\text { L'Aguilica y } \\
\text { su mirador }\end{array}$ \\
\hline
\end{tabular}




\begin{tabular}{|c|c|c|c|}
\hline \multirow[t]{6}{*}{$\begin{array}{c}\text { Túnel de } \\
\text { descarga } \\
\text { musealizado }\end{array}$} & $\begin{array}{c}\text { Búnker } \\
\text { Matalentisco } \\
\text { II }\end{array}$ & $\begin{array}{c}\text { Pava de la } \\
\text { Balsa }\end{array}$ & $\begin{array}{l}\text { Rincón del } \\
\text { Hornillo o de } \\
\text { los Casucos }\end{array}$ \\
\hline & Búnker Tébar & $\begin{array}{c}\text { Plaza de } \\
\text { España }\end{array}$ & $\begin{array}{l}\text { Sala museo } \\
\text { José Matrán }\end{array}$ \\
\hline & Casa grande & $\begin{array}{l}\text { Pilica del } \\
\text { Portazgo }\end{array}$ & \\
\hline & Casino & $\begin{array}{c}\text { Termas } \\
\text { romanas }\end{array}$ & \\
\hline & $\begin{array}{c}\text { Chimenea de } \\
\text { la fábrica de } \\
\text { la conservera } \\
\text { aguileña }\end{array}$ & $\begin{array}{c}\text { Torre de las } \\
\text { Palomas }\end{array}$ & \\
\hline & $\begin{array}{c}\text { Chimenea de } \\
\text { la fundición } \\
\text { de la Loma }\end{array}$ & & \\
\hline
\end{tabular}

Fuente: elaboración propia

TABLA 3

Figuras de protección que ostentan los PIDs

\begin{tabular}{|c|c|c|}
\hline Figura de protección & $\mathbf{N}$ & $\mathbf{\%}$ \\
\hline $\begin{array}{c}\text { Bien de interés cultural } \\
\text { grado 1 }\end{array}$ & 10 & $16,9 \%$ \\
\hline $\begin{array}{c}\text { Bienes catalogados por su relevancia cultural, } \\
\text { gratalogados por su relevancia cultural, } \\
\text { grado 2 }\end{array}$ & 29 & $49,2 \%$ \\
\hline $\begin{array}{c}\text { Bienes catalogados por su relevancia cultural, } \\
\text { grado 3 }\end{array}$ & 6 & $10,2 \%$ \\
\hline Ninguno & 11 & $18,6 \%$ \\
\hline Fuente:
\end{tabular}

Fuente: elaboración propia

Como se puede observar en la tabla 3, de los 59 PIDs seleccionados, principalmente, el 49,2\% posee la figura de "Bienes catalogados por su relevancia cultural, grado 1", seguida de un 18,6\% que no posee ninguna y un $16,9 \%$ que son considerados BIC.

TABLA 4

Figuras de protección de los entornos donde se localizan los PIDs

\begin{tabular}{|l|c|c|}
\hline Figura de protección & $\mathbf{N}$ & $\mathbf{\%}$ \\
\hline Espacios naturales protegidos (ENP) & 4 & $6,8 \%$ \\
\hline
\end{tabular}




\begin{tabular}{|l|c|c|}
\hline Red Natura 2000 & 8 & $13,6 \%$ \\
\hline Lugares de importancia comunitario (LIC) & 8 & $13,6 \%$ \\
\hline Zonas especiales de conservación (ZEC) & 0 & $0 \%$ \\
\hline Zonas de especial protección para las aves (ZEPA) & 5 & $8,5 \%$ \\
\hline Ninguno & 48 & $81,4 \%$ \\
\hline
\end{tabular}

Fuente: elaboración propia

En la tabla 4 se contempla como una gran mayoría de los PIDs $(81,4 \%)$ no se encuentran localizados en entornos con protecciones asignadas, si estando presente las figuras de Red Natura 2000 y LIC en el 13,6\% de los elementos patrimoniales.

TABLA 5

Indicador "Estado de conservación"

\begin{tabular}{|l|c|c|}
\hline \multicolumn{1}{|c|}{ Indicador } & $\mathbf{N}$ & $\mathbf{\%}$ \\
\hline $\begin{array}{l}\text { Fuertemente degradado: Se encuentra prácticamente destruido o con } \\
\text { deterioros importantes. }\end{array}$ & 6 & $10,2 \%$ \\
\hline $\begin{array}{l}\text { Alterado: Se encuentra con deterioros que impiden apreciar algunas } \\
\text { características de interés. }\end{array}$ & 3 & $5,1 \%$ \\
\hline $\begin{array}{l}\text { Favorable con alteración: presenta algunos deterioros que no afectan de } \\
\text { manera determinante al valor o interés del PID. }\end{array}$ & 9 & $15,2 \%$ \\
\hline Favorable: El PID se encuentra bien conservado, prácticamente íntegro. & 41 & $69,5 \%$ \\
\hline
\end{tabular}

Fuente: elaboración propia

Atendiendo al estado de conservación, la mayoría de los PIDs posee un estado de conservación favorable, $84,7 \%$, existiendo un $15,3 \%$ que se encuentra en estado fuertemente degradado o alterado (tabla 5). Por otro lado, en base al tipo de figura (tabla 6), los BIC son los elementos más fuertemente degradados y alterados $(66,7 \%)$ comparándolos porcentualmente con el resto de figuras. Asimismo, los bienes catalogados por su relevancia cultural son los que mejor se encuentran conservados en cuantía y en comparación porcentual con el resto.

TABLA 6

Estado de conservación atendiendo al tipo de protección

\begin{tabular}{|c|c|c|c|}
\hline INDICADOR & $\begin{array}{c}\text { Bien de interés } \\
\text { cultural }\end{array}$ & $\begin{array}{c}\text { Bienes catalogados por } \\
\text { su relevancia cultural }\end{array}$ & Bienes sin figura \\
\hline
\end{tabular}




\begin{tabular}{|l|c|c|c|c|c|c|}
\hline & Recuento & $\begin{array}{c}\% \text { de la } \\
\text { fila }\end{array}$ & Recuento & $\begin{array}{c}\text { \% de la } \\
\text { fila }\end{array}$ & Recuento & $\begin{array}{c}\% \text { de } \\
\text { la fila }\end{array}$ \\
\hline $\begin{array}{l}\text { Fuertemente degradado: Se encuentra } \\
\text { prácticamente destruido o con deterioros } \\
\text { importantes. }\end{array}$ & 4 & $66,7 \%$ & 2 & $33,3 \%$ & 0 & $0 \%$ \\
\hline $\begin{array}{l}\text { Alterado: Se encuentra con deterioros que } \\
\text { impiden apreciar algunas características de } \\
\text { interés. }\end{array}$ & 2 & $66,7 \%$ & 1 & $33,3 \%$ & 0 & $0 \%$ \\
\hline $\begin{array}{l}\text { Favorable con alteración: presenta algunos } \\
\text { deterioros que no afectan de manera } \\
\text { determinante al valor o interés del PID. }\end{array}$ & 2 & $22,2 \%$ & 7 & $77,8 \%$ & 0 & $0 \%$ \\
\hline $\begin{array}{l}\text { Favorable: El PID se encuentra bien } \\
\text { conservado, prácticamente íntegro. }\end{array}$ & 2 & $4,9 \%$ & 28 & $68,3 \%$ & 11 & $26,8 \%$ \\
\hline
\end{tabular}

Fuente: elaboración propia

TABLA 7

Indicador "Estacionalidad"

\begin{tabular}{|l|c|c|}
\hline \multicolumn{1}{|c|}{ Indicador } & $\mathbf{N}$ & $\mathbf{\%}$ \\
\hline El lugar no puede visitarse por restricciones legales. & 7 & $11,9 \%$ \\
\hline $\begin{array}{l}\text { El lugar puede visitarse en cualquier época del año, teniendo en cuenta } \\
\text { el horario establecido de visita o sin horario. }\end{array}$ & 45 & $76,2 \%$ \\
\hline $\begin{array}{l}\text { El lugar puede visitarse, teniendo en cuenta que se encuentra en un } \\
\text { entorno privado. }\end{array}$ & 7 & $11,9 \%$ \\
\hline
\end{tabular}

Fuente: elaboración propia

TABLA 8

Estacionalidad atendiendo al tipo de protección

\begin{tabular}{|l|c|c|c|c|c|c|}
\hline \multirow{2}{*}{ INDICADOR } & \multicolumn{2}{|c|}{$\begin{array}{c}\text { Bien de interés } \\
\text { cultural }\end{array}$} & \multicolumn{2}{c|}{$\begin{array}{c}\text { Bienes catalogados por } \\
\text { su relevancia cultural }\end{array}$} & \multicolumn{2}{|c|}{ Bienes sin figura } \\
\cline { 2 - 7 } & Recuento & $\begin{array}{c}\% \text { de la } \\
\text { fila }\end{array}$ & Recuento & $\%$ de la fila & Recuento & $\begin{array}{c}\text { \% de la } \\
\text { fila }\end{array}$ \\
\hline $\begin{array}{l}\text { El lugar no puede visitarse por } \\
\text { restricciones legales. }\end{array}$ & 2 & $28,6 \%$ & 5 & $71,4 \%$ & 0 & $0 \%$ \\
\hline $\begin{array}{l}\text { El lugar puede visitarse en cualquier } \\
\text { época del año, teniendo en cuenta el } \\
\text { horario establecido de visita o sin } \\
\text { horario. }\end{array}$ & 5 & $11,2 \%$ & 29 & $64,4 \%$ & 11 & $24,4 \%$ \\
\hline $\begin{array}{l}\text { El lugar puede visitarse, teniendo en } \\
\text { cuenta que se encuentra en un entorno } \\
\text { privado. }\end{array}$ & 3 & $42,9 \%$ & 4 & $57,1 \%$ & 0 & $0 \%$ \\
\hline
\end{tabular}

Fuente: elaboración propia

En cuanto a su estacionalidad para ser visitado (tabla 7), la mayoría de los puntos de interés $(76,2 \%)$ se puede visitar a lo largo del año sin horario y/o atendiendo al horario establecido de visita, y con igual resultado, un 11,9\% no puede ser visitado por restricciones legales y otro puede visitarse teniendo en cuenta que se encuentra ubicado en un entorno privado. Atendiendo al tipo de figura (tabla 8 ), un mayor porcentaje $(71,4 \%$ ) de bienes catalogados por su relevancia cultural no puede visitarse por restricciones legales, al igual teniendo en cuenta el horario establecido $(64,4 \%)$. Por otro lado, un $57,1 \%$ de los bienes catalogados por su relevancia cultural y un $42,9 \%$ de los BICs pueden visitarse teniendo en cuenta que se encuentran en un entorno privado.

TABLA 9

Indicador "Condiciones de observación" 


\begin{tabular}{|l|c|c|}
\hline \multicolumn{1}{|c|}{ Indicador } & N & $\%$ \\
\hline $\begin{array}{l}\text { Presencia de elementos que enmascaran fuertemente las características } \\
\text { de interés }\end{array}$ & 5 & $8,5 \%$ \\
\hline $\begin{array}{l}\text { Presencia de elementos que enmascaran el PID y que impiden apreciar } \\
\text { algunas características de interés. }\end{array}$ & 5 & $8,5 \%$ \\
\hline $\begin{array}{l}\text { Con algún elemento que impide observar el PID en su integridad pero } \\
\text { que no afectan a la apreciación de sus características de interés. }\end{array}$ & 10 & $16,9 \%$ \\
\hline Perfectamente observable en su integridad y con facilidad. & 39 & $66,1 \%$ \\
\hline
\end{tabular}

Fuente: elaboración propia

TABLA 10

Condiciones de observación atendiendo al tipo de protección

\begin{tabular}{|c|c|c|c|c|c|c|}
\hline \multirow[t]{2}{*}{ INDICADOR } & \multicolumn{2}{|c|}{$\begin{array}{l}\text { Bien de interés } \\
\text { cultural }\end{array}$} & \multicolumn{2}{|c|}{$\begin{array}{c}\text { Bienes catalogados } \\
\text { por su relevancia } \\
\text { cultural }\end{array}$} & \multicolumn{2}{|c|}{ Bienes sin figura } \\
\hline & Recuento & $\begin{array}{c}\% \text { de la } \\
\text { fila }\end{array}$ & Recuento & $\begin{array}{c}\text { \% de la } \\
\text { fila }\end{array}$ & Recuento & $\begin{array}{c}\% \text { de la } \\
\text { fila }\end{array}$ \\
\hline $\begin{array}{llr}\text { Presencia de elementos } & \text { que } \\
\text { enmascaran fuertemente } & \text { las } \\
\text { características de interés. } & \\
\end{array}$ & 3 & $60 \%$ & 2 & $40 \%$ & 0 & $0 \%$ \\
\hline $\begin{array}{l}\text { Presencia de elementos que } \\
\text { enmascaran el PID y que impiden } \\
\text { apreciar algunas características de } \\
\text { interés. }\end{array}$ & 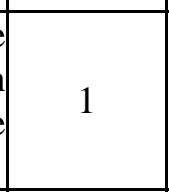 & $20 \%$ & 4 & $80 \%$ & 0 & $0 \%$ \\
\hline $\begin{array}{l}\text { Con algún elemento que impide } \\
\text { observar el PID en su integridad pero } \\
\text { que no afectan a la apreciación de sus } \\
\text { características de interés. }\end{array}$ & 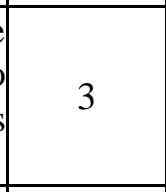 & $30 \%$ & 7 & $70 \%$ & 0 & $0 \%$ \\
\hline $\begin{array}{l}\text { Perfectamente observable en su } \\
\text { integridad y con facilidad. }\end{array}$ & 3 & $7,7 \%$ & 25 & $64,1 \%$ & 11 & $28,2 \%$ \\
\hline
\end{tabular}

Fuente: elaboración propia

Las tablas 9 y 10 reflejan como el $66,1 \%$ de los puntos puede observarse perfectamente en su integridad y con facilidad, siendo la mayoría. De éstos, el $64,1 \%$ son bienes catalogados por su relevancia cultura, seguido de un $28,8 \%$ de bienes sin figura Por otro lado, el $16,9 \%$ posee elementos que impiden observar el lugar en su integridad pero que no afecta a la apreciación de sus características, siendo un $70 \%$ de bienes catalogados por su relevancia cultural y un $30 \%$ los BICs. El resto de puntos posee elementos que enmascaran su observación en mayor o menor medida.

TABLA 11

Indicador "Peligrosidad del terreno"

\begin{tabular}{|l|c|c|}
\hline \multicolumn{1}{|c|}{ Indicador } & $\mathbf{N}$ & $\mathbf{\%}$ \\
\hline $\begin{array}{l}\text { Existen elementos que hacen que el lugar sea altamente peligroso, aun } \\
\text { tomando medidas de seguridad y precaución. }\end{array}$ & 1 & $1,7 \%$ \\
\hline
\end{tabular}




\begin{tabular}{|l|c|c|}
\hline $\begin{array}{l}\text { Existen elementos que hacen del lugar parcialmente peligroso, aun } \\
\text { tomando medidas de seguridad. }\end{array}$ & 2 & $3,4 \%$ \\
\hline $\begin{array}{l}\text { Existen elementos que hace que el lugar sea parcialmente peligroso solo } \\
\text { si no se toman medidas de seguridad. }\end{array}$ & 2 & $3,4 \%$ \\
\hline No existe peligrosidad asociada al lugar. & 54 & $91,5 \%$ \\
\hline
\end{tabular}

Fuente: elaboración propia

TABLA 12

Peligrosidad del terreno atendiendo al tipo de protección

\begin{tabular}{|l|c|c|c|c|c|c|}
\hline \multirow{2}{*}{ INDICADOR } & \multicolumn{2}{|c|}{$\begin{array}{c}\text { Bien de interés } \\
\text { cultural }\end{array}$} & $\begin{array}{c}\text { Bienes catalogados } \\
\text { por su relevancia } \\
\text { cultural }\end{array}$ & \multicolumn{2}{|c|}{ Bienes sin figura } \\
\cline { 2 - 7 } & Recuento & $\begin{array}{c}\text { \% de la } \\
\text { fila }\end{array}$ & Recuento & $\begin{array}{c}\text { \% de la } \\
\text { fila }\end{array}$ & Recuento & $\begin{array}{c}\text { \% de la } \\
\text { fila }\end{array}$ \\
\hline $\begin{array}{l}\text { Existen elementos que hacen que el } \\
\text { lugar sea altamente peligroso, aun } \\
\text { tomando medidas de seguridad y } \\
\text { precaución. }\end{array}$ & 1 & $100 \%$ & 0 & $0 \%$ & 0 & $0 \%$ \\
\hline $\begin{array}{l}\text { Existen elementos que hacen del } \\
\text { lugar parcialmente peligroso, aun } \\
\text { tomando medidas de seguridad. }\end{array}$ & 2 & $100 \%$ & 0 & $0 \%$ & 0 & $0 \%$ \\
\hline $\begin{array}{l}\text { Existen elementos que hace que el } \\
\text { lugar sea parcialmente peligroso } \\
\text { solo si no se toman medidas de } \\
\text { seguridad. }\end{array}$ & 0 & $0 \%$ & 1 & $50 \%$ & 1 & $50 \%$ \\
\hline $\begin{array}{l}\text { No existe peligrosidad asociada al } \\
\text { lugar. }\end{array}$ & 7 & $13 \%$ & 37 & $68,5 \%$ & 10 & $18,5 \%$ \\
\hline
\end{tabular}

Fuente: elaboración propia

En la tabla 11 se puede observar como una gran mayoría de los PIDs $(91,5 \%)$ se encuentra en un terreno sin peligrosidad, existiendo por lo tanto un 8,5\% que se localiza en un entorno peligroso. De éstos últimos, un BIC se encuentra en un lugar altamente peligroso, dos BIC en espacios parcialmente peligrosos aun tomando medidas, y por último, dos lugares parcialmente peligrosos si no se toman medidas preventivas: un bien catalogado por su relevancia cultural y un bien sin figura (tabla 12).

TABLA 13

Indicador "Accesibilidad"

\begin{tabular}{|l|c|c|}
\hline \multicolumn{1}{|c|}{ Indicador } & N & $\%$ \\
\hline Sin acceso directo por camino transitable. & 7 & $11,9 \%$ \\
\hline
\end{tabular}




\begin{tabular}{|l|c|c|}
\hline $\begin{array}{l}\text { Con acceso directo por camino transitable para personas que no } \\
\text { presentan movilidad reducida. }\end{array}$ & 16 & $27,1 \%$ \\
\hline $\begin{array}{l}\text { Con acceso directo por camino transitable para personas con movilidad } \\
\text { reducida y resto de usuarios. }\end{array}$ & 36 & $61 \%$ \\
\hline
\end{tabular}

Fuente: elaboración propia

TABLA 14

Accesibilidad atendiendo al tipo de protección

\begin{tabular}{|c|c|c|c|c|c|c|}
\hline \multirow[t]{2}{*}{ INDICADOR } & \multicolumn{2}{|c|}{$\begin{array}{l}\text { Bien de interés } \\
\text { cultural }\end{array}$} & \multicolumn{2}{|c|}{$\begin{array}{c}\text { Bienes catalogados } \\
\text { por su relevancia } \\
\text { cultural }\end{array}$} & \multicolumn{2}{|c|}{ Bienes sin figura } \\
\hline & Recuento & $\begin{array}{c}\text { \% de la } \\
\text { fila }\end{array}$ & Recuento & $\begin{array}{c}\text { \% de la } \\
\text { fila }\end{array}$ & Recuento & $\begin{array}{c}\% \text { de la } \\
\text { fila }\end{array}$ \\
\hline $\begin{array}{l}\text { Sin acceso directo por camino } \\
\text { transitable. }\end{array}$ & 3 & $42,9 \%$ & 3 & $42,9 \%$ & 1 & $14,3 \%$ \\
\hline $\begin{array}{l}\text { Con acceso directo por camino } \\
\text { transitable para personas que no } \\
\text { presentan movilidad reducida. }\end{array}$ & 2 & $12,5 \%$ & 12 & $75 \%$ & 2 & $12,5 \%$ \\
\hline $\begin{array}{l}\text { Con acceso directo por camino } \\
\text { transitable para personas con } \\
\text { movilidad reducida y resto de } \\
\text { usuarios. }\end{array}$ & 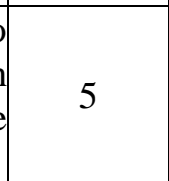 & $13,9 \%$ & 23 & $63,9 \%$ & 8 & $22,2 \%$ \\
\hline
\end{tabular}

Fuente: elaboración propia

En cuanto a la accesibilidad (tabla 13 y tabla 14), al 61\% de los PIDs se accede por camino transitable para personas con movilidad reducida y resto de usuarios (sobre todo en bienes catalogados por su relevancia cultural, $63,9 \%$ ), seguido de un $27,1 \%$ por camino para personas que no presentan movilidad reducida (principalmente, bienes catalogados por su relevancia cultural con un $75 \%$ ), y por último, un $11,9 \%$ no posee acceso por camino transitable (por igual en BICs y bienes catalogados por su relevancia cultural, $42,9 \%$ ).

TABLA 15

Indicador "Información disponible"

\begin{tabular}{|l|c|c|}
\hline \multicolumn{1}{|c|}{ Indicador } & $\mathbf{N}$ & $\boldsymbol{\%}$ \\
\hline El PID no posee ningún tipo de información “in situ”. & 44 & $74,6 \%$ \\
\hline El PID posee información “in situ”. & 15 & $25,4 \%$ \\
\hline
\end{tabular}

Fuente: elaboración propia

TABLA 16

Información disponible antendiendo al tipo de protección

INDICADOR

Bien de interés cultural

Bienes catalogados

por su relevancia Bienes sin figura cultural 


\begin{tabular}{|c|c|c|c|c|c|c|}
\hline & Recuento & $\begin{array}{c}\% \text { de la } \\
\text { fila }\end{array}$ & Recuento & $\begin{array}{c}\text { \% de la } \\
\text { fila }\end{array}$ & Recuento & $\begin{array}{c}\% \text { de la } \\
\text { fila }\end{array}$ \\
\hline $\begin{array}{l}\text { El PID no posee ningún tipo de } \\
\text { información "in situ". }\end{array}$ & 6 & $13,6 \%$ & 30 & $68,2 \%$ & 8 & $18,2 \%$ \\
\hline El PID posee información “in situ”. & 4 & $26,7 \%$ & 8 & $53,3 \%$ & 3 & $20 \%$ \\
\hline
\end{tabular}

Fuente: elaboración propia

En cuanto al último indicador, información disponible (tabla 15 y tabla 16), el 74,6\% de los PIDs no posee ningún tipo de información "in situ", siendo un $68,2 \%$ de bienes catalogados por su relevancia cultural, seguido de un $18,2 \%$ por parte de los bienes sin figura. Por otro lado, un $25,4 \%$ si poseen información, principalmente un $53,3 \%$ de bienes catalogados por su relevancia cultural y los BICs con un 26,7\%.

\section{DISCUSIÓN Y CONCLUSIONES}

El objetivo del presente trabajo es identificar y valorar los puntos de interés didáctico existentes en Águilas.

El municipio posee al menos 38 PIDs que ostentan la figura de bienes catalogados por su relevancia cultural y solo 10 con la figura de BIC, cuantías contrarias al de la figura más difundida en los medio de comunicación. Siendo la BIC la figura más popular, a la vez que la más relevante a nivel de protección patrimonial en España, eco de esto se puede encontrar diversas noticias de Águilas que se han publicado relacionadas con el BIC (EUROPAPRESS, 25 de septiembre de 2009; LA OPINIÓN DE MURCIA, 26 de abril de 2012) y no con el resto de figuras. Asimismo, en relación a las figuras asignadas al entorno, la mayoría de PIDs se encuentra en lugares reconocidos en la Red Natura 2000, LIC y ZEPA.

Por otra parte, un $40 \%$ de los PIDs no presenta un estado íntegramente conservado, dato que confronta con la Ley de Patrimonio Histórico Español (JEFATURA DEL ESTADO, 29 de junio de 1985), donde en su artículo segundo se contempla: "son deberes y atribuciones esenciales de la Administración del Estado garantizar la conservación del patrimonio histórico español, así como promover el enriquecimiento del mismo y fomentar y tutelar el acceso de todos los ciudadanos a los bienes comprendidos en él" (p. 20342), y en el artículo séptimo:

Los ayuntamientos cooperarán con los Organismos competentes para la ejecución de esta Ley en la conservación y custodia del patrimonio histórico español comprendido en su término municipal, adoptando las medidas oportunas para evitar su deterioro, pérdida o destrucción (20343).

En cuanto a su visita, una cuarta parte de los PIDs no puede visitarse por restricciones legales, o sí, pero teniendo en cuenta que se encuentra en un entorno privado, en este caso, las personas o entidades que poseen el patrimonio cultural están obligadas a abrir al público los bienes al menos durante cuatro días al mes, tal y como indica CARRETÓN (2016), debiendo informar sobre cuáles son esos días y las horas de visita. En relación a la accesibilidad, casi el $40 \%$ de los PIDs no es accesible para personas con movilidad reducida, aun existiendo el derecho de disfrute del patrimonio para todas las personas mediante una accesibilidad universal, como se recoge en la Ley de Igualdad de Oportunidades, no Discriminación y Accesibilidad Universal (JEFATURA DEL ESTADO, 03 de diciembre de 2003).

En cuanto a la información que se puede encontrar junto al patrimonio a través de paneles interpretativos, el 74,6\% de los PIDs analizados no posee ningún tipo de información "in situ". Se puede intuir en base al proceso de investigación llevado a cabo, que esto puede ser debido a varias razones: el coste que conlleva la edición, instalación y mantenimiento de este tipo de infraestructuras; que los PIDs se encuentren en entornos privados o que sean de titularidad privada, dificultando los tramites o imposibilitando su colocación; que el terreno no sea adecuado para la instalación, etc.; o directamente la dejadez de los organismos encargados de esa función. Ante estos diversos problemas y la ausencia de paneles interpretativos, una interesante solución podría ser la edición de una guía de puntos de interés didáctico y/o turístico, como ya han hecho otros autores (CORVEA et al., 2006), pudiéndose observar un ejemplo de ficha de un PID de 
Águilas en la figura 1. Esta guía podría estar disponible en formato papel y/o digital, y ser utilizada en cualquier momento y lugar.

FIGURA 1

Ficha ejemplo de un PID

PID-01. CASTILLO DE CHUECOS

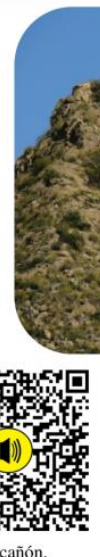

cañon

Toda h coscaleras o saeteras abiertas en el muro de la torre.

Toda la construcción fue levantada mediante la técnica del tapial, que cosite on construir con tierra arcillosa, compactada a golpes y empleando un encofrado deslizante para contenerla.

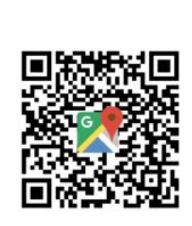

para las Aves (ZEPA).

ituado a 777 metros de altitud en la Sierra de Almenara, está datado en los siglos XI y XII. De origen islámico, daba protección y cobertura a la población dedicada a labor

El recinto presenta un perímetro de forma rectangular, en el extremo norte se encuentra un gran torreón de planta cuadra que conserva unos 6.5 metros de altura, mientras que en lado sur hay un aljibe de grande dimensiones, planta rectangular y Áreas educativa:
INDICADORES

Ubicación: Sierra de Almenara

Tipo de observación y acceso: Desde el interior (no existe senda, no apto para movilidad reducida) y exterior lejana (a pie o en vehículo/ no apto para movilidad reducida).

Figuras asignadas: Bien de Interés Cultural.

Figuras asignadas al entorno: Red Natura 2000, Lugares de

Importancia Comunitaria (LIC) y Zona de Especial Protección

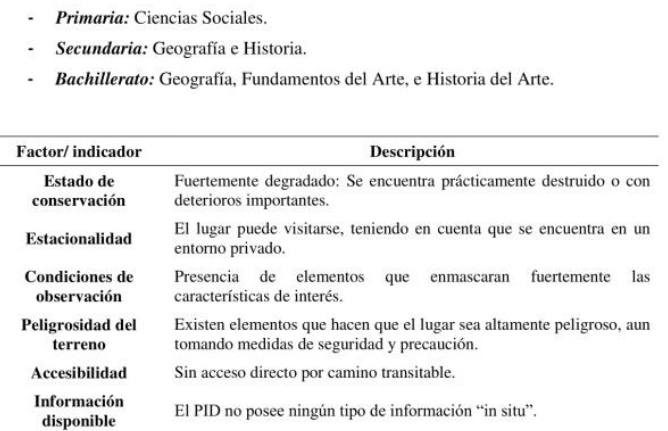

- Primaria: Ciencias Sociales.

Secundaria: Geografía e Histori

Fuente: elaboración propia

Por otro lado y en la línea de acercamiento del patrimonio a los ciudadanos, la integración de recursos tecnológicos de información geográfica favorece tal proceso, ya que a través ellas, se puede recopilar una gran cantidad de información, gestionando o analizando la información georeferenciada, a través de imágenes topográficas o vista satélite, medir la distancia entre dos o varios puntos, diseñar un itinerario o ruta, orientarse, obtener las coordenadas geográficas, etc. (BUZO, 2015; MARTÍN, NIETO y BUZO, 2016), un ejemplo de aplicación tecnológica y que se propone en este trabajo es MyMaps (figura 2).

FIGURA 2

Inventario de PIDs en la plataforma MyMaps 


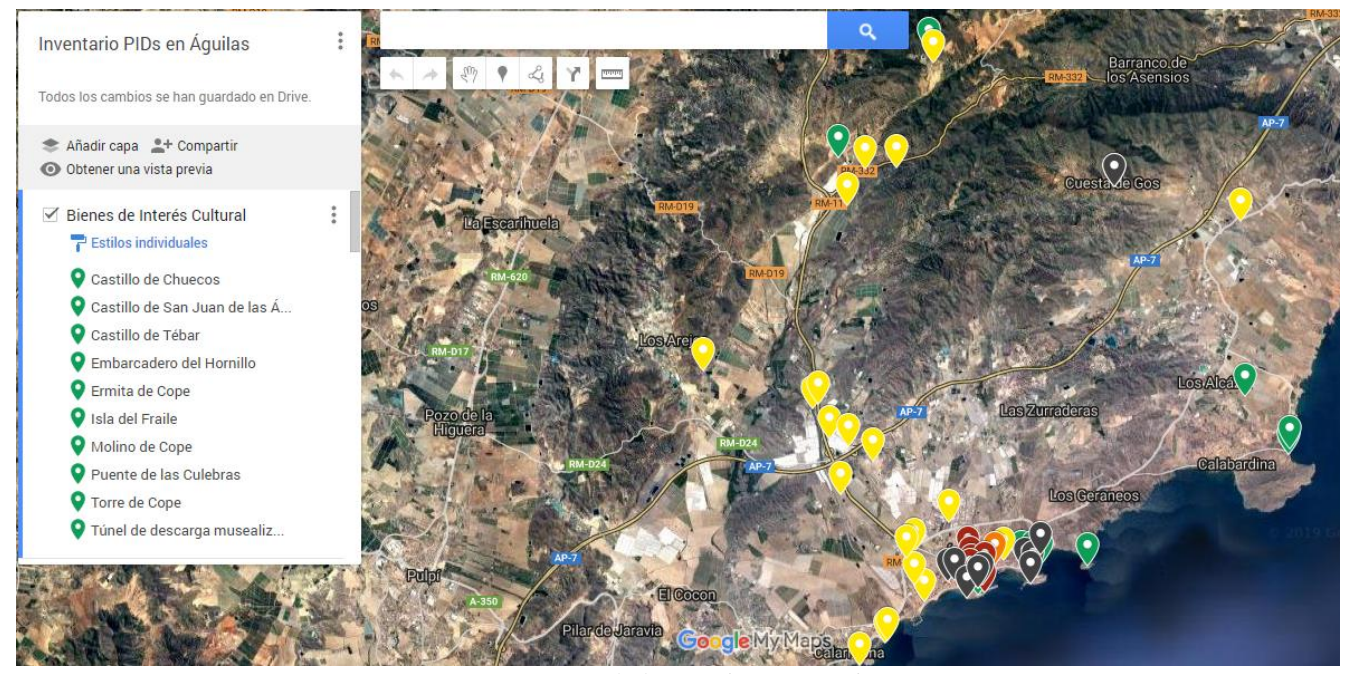

Fuente: elaboración propia

Por último, se puede concluir que Águilas posee, al menos, un total de 59 PIDs: 10 BICs, 38 bienes catalogados por su relevancia cultura y 11 bienes sin figura asignada. De éstos, casi un tercio de los PIDs se encuentran en un estado de conservación desfavorable, siendo principalmente los BIC. La mayoría del patrimonio puede visitarse, aunque existe una parte que por restricciones legales o porque se encuentra en un entorno privado se debe comunicar el deseo de visita para evitar posibles conflictos con el propietario. Por otra parte, el 60\% de los PIDS es accesible para personas con movilidad reducida, frente a un $40 \%$ que no, existiendo una limitación de accesibilidad para un importante sector de la población (discapacitados, ancianos, etc.). Por último, un casi $75 \%$ de los PIDs no posee ningún tipo de información "in situ", limitando así, el conocimiento de su historia, datos de interés, etc. al visitante y la valoración de otras parte de él.

Para terminar se valora y propone la edición de una guía de puntos de interés didáctico y/o turístico, y un inventario de los PIDs a través de la plataforma MyMaps, con el fin de acercar el patrimonio existente en Águilas a los ciudadanos. Siendo además necesarias otras actuaciones en base a los resultados obtenidos en el presente trabajo, en relación a la accesibilidad, estacionalidad, peligrosidad, etc.

\section{REFERENCIAS BIBLIOGRÁFICAS}

AYUNTAMIENTO DE ÁGUILAS (s.f.a): Catálogo etnográfico. Recuperado de https://www.ayuntamientodeaguilas.org/pgou/04 CATALOGO/etnografico/1.pdf

AYUNTAMIENTO DE ÁGUILAS (s.f.b): Catálogo de edificios protegidos. Recuperado de https://www.ayuntamientodeaguilas.org/pgou/04 CATALOGO/edificios/1.pdf

AYUNTAMIENTO DE ÁGUILAS (s.f.c): Catálogo arqueológico y paleontológico. Recuperado de https://www.ayuntamientodeaguilas.org/pgou/04_CATALOGO/arqueologico/1.pdf

AYUNTAMIENTO DE ÁGUILAS (s.f.d): Catálogo de árboles singulares y monumentales. Recuperado de https://www.ayuntamientodeaguilas.org/pgou/04_CATALOGO/arboles/1.pdf AYUNTAMIENTO DE ÁGUILAS (s.f.e): Documento III: Catálogo de elementos protegidos. Recuperado de https://www.ayuntamientodeaguilas.org/lccloud/adjuntos/mcope/general/5.pdf

BALLART, J. (2001): El patrimonio histórico y arqueológico. Valor y uso. Ariel, Barcelona.

BUZO, I. (2015): "Los SIG como herramienta para el estudio del paisaje cultural". Iber: Didáctica de las Ciencias Sociales, Geografía e Historia, vol. 81, p. 37-40. Recuperado de https://www.grao.com/ca/producte/materiales-y-recursos-los-sig-como-herramienta-para-elestudio-del-paisaje-cultural

CARRETÓN, A. (27 de marzo de 2016): Obligaciones a la propiedad de bienes culturales. Recuperado de https://patrimoniointeligente.com/obligaciones-la-propiedad-bienesculturales/ 
COMUNIDAD AUTÓNOMA DE LA REGIÓN DE MURCIA (12 de abril de 2007): Ley de Patrimonio Cultural de la Comunidad Autónoma de la Región de Murcia [Ley 4 de 2007]. Recuperado de https://www.boe.es/buscar/pdf/2008/BOE-A-2008-12526-consolidado.pdf

CONSEJO DE GOBIERNO (06 de septiembre de 2014): Decreto por el que se establece el currículo de la Educación Primaria en la Comunidad Autónoma de la Región de Murcia. [Decreto 198 de 2014]. Recuperado de https://www.borm.es/borm/documento?obj=anu\&id=713895

CONSEJO DE GOBIERNO (03 de septiembre de 2015a): Decreto por el que se establece el currículo de la Educación Secundaria Obligatoria en la Comunidad Autónoma de la Región de Murcia. [Decreto 220 de 2015]. Recuperado de https://www.borm.es/borm/documento?obj=anu\&id=735576

CONSEJO DE GOBIERNO (03 de septiembre de 2015b): Decreto por el que se establece el currículo del Bachillerato en la Comunidad Autónoma de la Región de Murcia. [Decreto 221 de 2015]. Recuperado de https://www.borm.es/borm/documento?obj=anu\&id=735577

CORVEA, J. L. (2001): Utilización didáctica de la cartografía temática ambiental de un sector del Norte de La Comunidad de Madrid. Tesis Doctoral. Universidad de Alcalá, Madrid.

CORVEA, J. L., BUSTAMANTE, I., GARCÍA-HIDALGO, J. F., SANZ, J. M. y MATEOS, J. (2006): Guía de Puntos de Interés Didáctico del Norte de la Comunidad de Madrid. Universidad de Alcalá de Henares, Madrid. Recuperado de https://www.madrimasd.org/cienciaysociedad/patrimonio/rutas/geologicas/PuntosInteres/co mun/documentos/Guia_puntos_interes.pdf

CRESPO, J. M., GÓMEZ, M. L. y CRUZ, L. A. (2018): "Una aproximación a los Parques Nacionales y sus paisajes a través de itinerarios didácticos". Revista Espacio, Tiempo y Forma, vol. 11, p. 121-140. Doi: https://doi.org/10.5944/etfvi.11.2018.22359

Cuenca, J. M. (2014): "El papel del patrimonio en los centros educativos: hacia la socialización patrimonial". Tejuelo, vol. 19, p. 76-96. Recuperado de https://mascvuex.unex.es/revistas/index.php/tejuelo/article/view/2566

CUENCA, J. M., ESTEPA, J. y MARTÍN, M. J. (2011): "El patrimonio cultural en la educación reglada". Revista Patrimonio Cultural de España, $\mathrm{n}^{\circ}$ 5, p. 45-58. Recuperado de http://rabida.uhu.es/dspace/bitstream/handle/10272/9437/El_patrimonio_cultural.pdf?sequen ce $=2$

EUROPAPRESS (25 de septiembre de 2009): MURCIA.-Consejo.- El embarcadero del Hornillo de Águilas recibe la declaración de Bien de Interés Cultural. EuropaPress. Recuperado de https://www.europapress.es/cultura/noticia-murcia-consejo-embarcadero-hornillo-aguilasrecibe-declaracion-bien-interes-cultural-20090925140249.html

GARCÍA, A. L. (1994): "Los Itinerarios Didácticos, una de las claves para la Enseñanza y Comprensión de la Geografía". Revista IBER, $\mathrm{n}^{\circ}$ 1, p. 117-126. Recuperado de https://www.grao.com/es/producto/los-procedimientos-en-historia-ib001

INSA, Y. (2002): "Itinerarios urbanos, recursos y materiales didácticos para explicar la ciudad". Revista Íber, vol. 32, p. 89-95. Recuperado de https://www.grao.com/es/producto/itinerariosurbanos-recursos-y-materiales-didacticos-para-explicar-la-ciudad

JEFATURA DEL ESTADO (29 de junio de 1985): Ley de Patrimonio Histórico Español. [Ley 16 de 1985]. Recuperado de https://www.boe.es/buscar/act.php?id=BOE-A-1985-12534

JEFATURA DEL ESTADO (03 de diciembre de 2003): Ley de igualdad de oportunidades, no discriminación y accesibilidad universal de las personas con discapacidad. [Ley 51 de 2003]. Recuperado de https://www.boe.es/eli/es/1/2003/12/02/51

JEFATURA DEL ESTADO (14 de diciembre de 2007): Ley Orgánica complementaria de la Ley para el desarrollo sostenible del medio rural [Ley 16 de 2007]. Recuperado de https://www.boe.es/boe/dias/2007/12/14/pdfs/A51275-51327.pdf

LA OPINIÓN DE MURCIA (26 de abril de 2012): La Isla del Fraile, declarada Bien de Interés Cultural. La Opinión de Murcia. Recuperado de https://www.laopiniondemurcia.es/municipios/2012/04/26/isla-fraile-declarada-interescultural/400481.html 
LÓPEZ, F. y SEGURA, J. A. (2013): "Los itinerarios didácticos: un recurso interdisciplinar y vertebrador del curriculum". Espiral. Cuadernos del Profesorado, vol. 6, n 12, p. 15-31. Doi: http://dx.doi.org/10.25115/ecp.v6i12

MAILLARD, C. (2012): Construcción social del patrimonio. "Hecho en Chile: Reflexiones en torno al patrimonio cultural". En Marsal, (Ed.). Santiago de Chile, Chile: Ediciones Consejo Nacional de las Artes y la Cultura, pp.17-29.

MARTÍN, S. (2016): Propuesta metodológica para el diseño de itinerarios didácticos de Ciencias de la Naturaleza. Trabajo Fin de Máster. Universidad Autónoma de Madrid, Madrid. Recuperado

de https://repositorio.uam.es/bitstream/handle/10486/671862/DE_14_11.pdf?sequence=1\&isAll owed=y

MARTÍN, J. M., NIETO, A. y BUZO, I. (2016): Los SIG aplicados a la enseñanza de la Geografía en $1^{\circ}$ de Educación Secundaria. "Tecnologías de la Información Geográfica en el Análisis Espacial. Aplicaciones en los Sectores Público, Empresarial y Universitario". En A. Nieto Masot (Coord.). Universidad de Extremadura, Grupo de Investigación en Desarrollo Sostenible y Planificación Territorial: Universidad de Extremadura, Grupo de Investigación Geo-Ambiental, pp. 141-160. Recuperado de https://dialnet.unirioja.es/descarga/libro/667265.pdf

MARTÍNEZ, F. J. y GARCÍA, A. J. (2008): "Itinerarios didácticos por Fuente Álamo (Murcia), una estrategia educativa de innovación en el proceso de enseñanza-aprendizaje en Educación Secundaria". Espiral. Cuadernos del Profesorado, vol. 1, $\mathrm{n}^{\circ}$ 1, p. 1-9. Doi: http://ojs.ual.es/ojs/index.php/ESPIRAL/issue/view/90

MÍNGUEZ, M. C. (2010): "El paisaje como objeto de estudio de la Geografía. Un itinerario didáctico en el marco de la semana de la ciencia de la Comunidad de Madrid". Didáctica Geográfica, $\mathrm{n}^{\mathrm{o}} \quad 11, \quad$ p. 37-62. Recuperado de http://www.agegeografia.es/didacticageografica/index.php/didacticageografica/article/view/40

MINISTERIO DE EDUCACIÓN, CULTURA Y DEPORTE (29 de enero de 2015): Orden por la que se describen las relaciones entre las competencias, los contenidos y los criterios de evaluación de la educación primaria, la educación secundaria obligatoria y el bachillerato. [Orden ECD 65 de 2015]. Recuperado de https://www.boe.es/boe/dias/2015/01/29/pdfs/BOEA-2015-738.pdf

REGIÓN DE MURCIA DIGITAL. (s. f.): Municipio de Águilas. Recuperado de http://www.regmurcia.com/servlet/s.Sl?sit=a,64

SERRANO DE LA CRUZ, M. A., GARCÍA, J. L. y JEREZ, O. (2017): "Propuesta preliminar para la identificación de lugares de interés didáctico en espacios naturales protegidos". Didáctica Geográfica, vol. 17, p. 159-176. Recuperado de http://www.agegeografia.es/didacticageografica/index.php/didacticageografica/article/view/364

TORT, J. (2004): "El paisaje como pedagogía del territorio". Didáctica Geográfica, nº 6, p. 133153. Recuperado de http://www.agegeografia.es/didacticageografica/index.php/didacticageografica/article/view/189

UNESCO (2014): Manual Metodológico. Indicadores UNESCO de cultural para el desarrollo. UNESCO, París. Recuperado de https://es.unesco.org/creativity/sites/creativity/files/iucd_manual_metodologico_1.pdf 